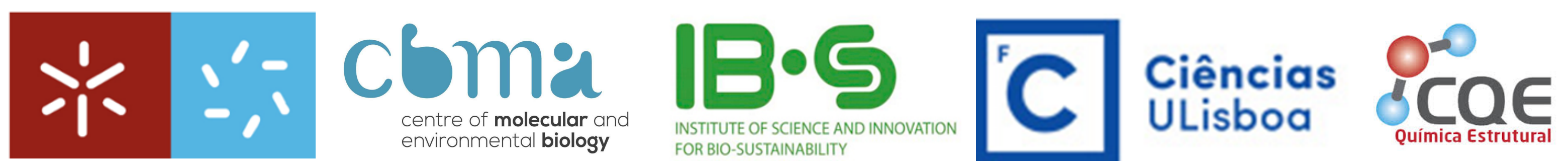

\section{Ruthenium-based agents as promising metallodrugs to fight colorectal cancer}

\author{
AR Brás ${ }^{1,2,3}$, P Fernandes ${ }^{1,2}$, T Moreira, A Valente $^{3^{*}}$, A Preto $^{1,2^{*}}$ \\ ${ }^{1}$ CBMA-Centre of Molecular and Environmental Biology, University of Minho, Braga, Portugal; 2IB-S - Institute of Science and Innovation for Bio-Sustainability, University of Minho, Braga, \\ Portugal; ${ }^{3}$ CQE - Centro de Química Estrutural, Faculty of Science of University of Lisbon, Lisbon, Portugal \\ ${ }^{*}$ Co-senior authorship
}

\section{BACKGROUND}

Colorectal cancer (CRC) is one of the most lethal cancers worldwide ${ }^{1}$. CRC therapy has limited chemotherapeutic agents available ${ }^{2}$. Ruthenium (Ru) drugs have arisen as one of the most promising metallodrugs with features that increase their specificity and selectivity toward cancer cells ${ }^{3}$. In this work, we studied three new Ru-cyclopentadienyl (RuCp) conjugates (LCR134, PMC78 and LCR220) in CRC cells (Figure 1). These conjugates comprise a ruthenium cytotoxic core with anticancer properties (LCR134, PMC78 and LCR220), a biodegradable polymer chain (PMC78 and LCR220) allowing the accumulation by the enhanced permeability and retention effect (EPR), and a biotin molecule (LCR134 and LCR220) allowing a selective accumulation in malignant tissues ${ }^{4,5}$. The addition of biotin to the ruthenium cytotoxic core also constitutes an approach to increase the targeting to cancer cells, due to the overexpression of vitamin receptors in these cells. The conjugation of these characteristics make these complexes more specific, efficient and potentially selective to target cancer cells.

AIM

To unveil the effect and mechanism of action of RuCp agents in CRC cells harboring different oncogenic mutations (SW480 ${ }^{\mathrm{KRAS}}$, RKOBRAF) in comparison to normal colon cells (NCM460).

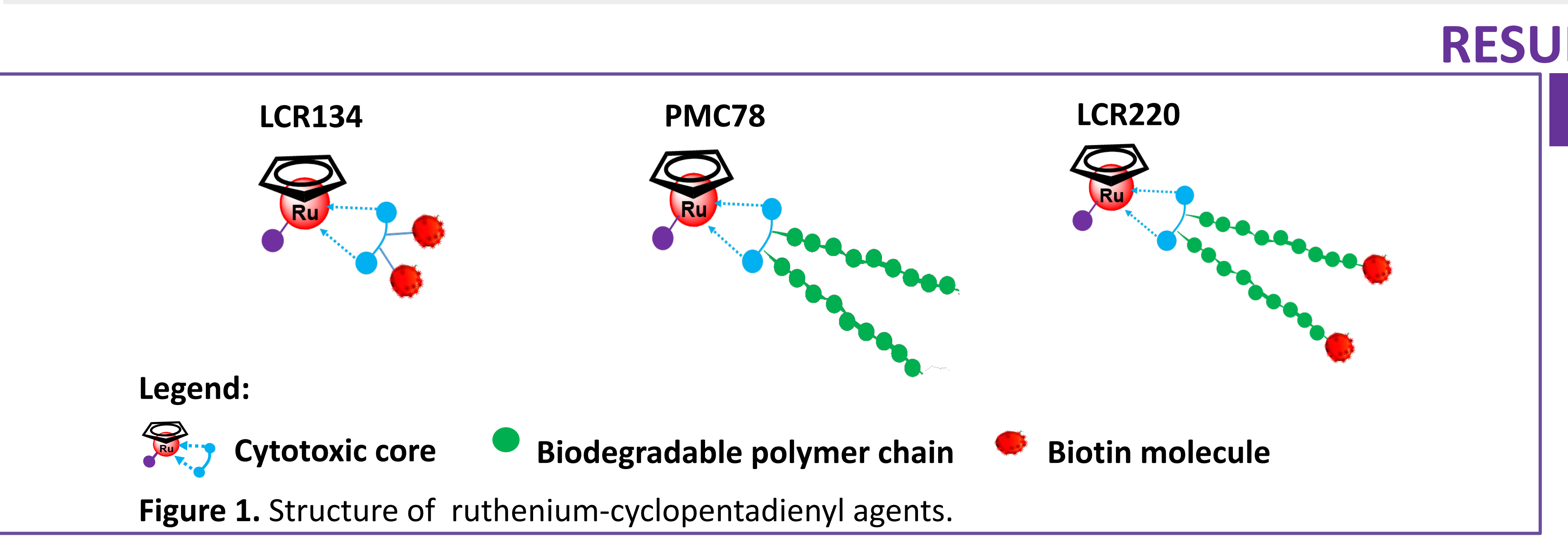

Ru agents are more cytotoxic and potentially selective to CRC cells

\begin{tabular}{|cccc|c|c|}
\hline \multicolumn{5}{|c|}{ Table 1. Determination of the $\mathrm{IC}_{50}$ values of Ru agents by Sulforhodamine B. } \\
\hline Compound & $\mathrm{IC}_{50}(\mu \mathrm{M})$ & $\mathrm{IC}_{50}(\mu \mathrm{M})$ & $\mathrm{IC}_{50}(\mu \mathrm{M})$ & $\mathrm{SW} 480$ & $\mathrm{RKO}$ \\
\hline LCR134 & 14,1 & 7,7 & 57,2 & 4,1 & 7,4 \\
\hline PMC78 & 6,0 & 4,0 & 15,0 & 2,5 & 3,8 \\
\hline LCR220 & 1,8 & 2,8 & 4,3 & 2,4 & 1,5 \\
\hline
\end{tabular}

Ru agents decrease the clonogenic ability of CRC cells RKOBRAF

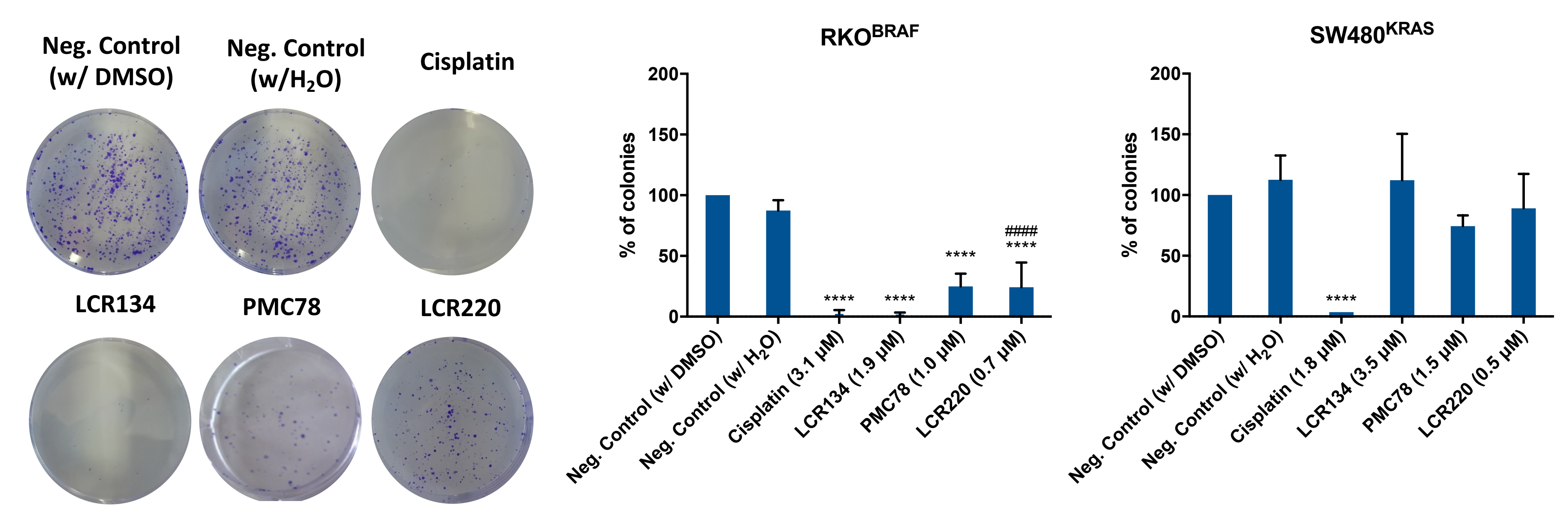

Figure 2. Representative images of RKO and graphics of colony formation ability of RKO and SW480 cells. Ru agents induce alterations in cell cycle of CRC cells

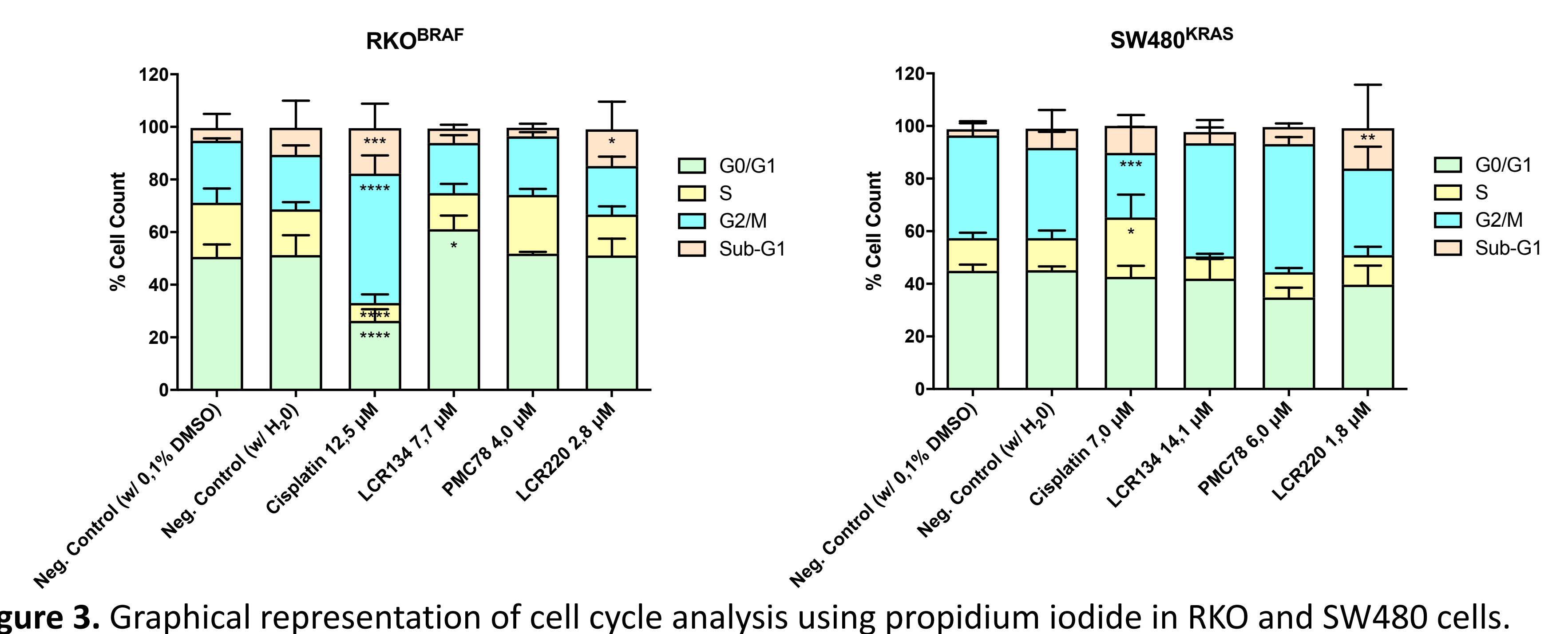

CONCLUSION
Ru agents induce apoptosis at high concentrations in CRC cells

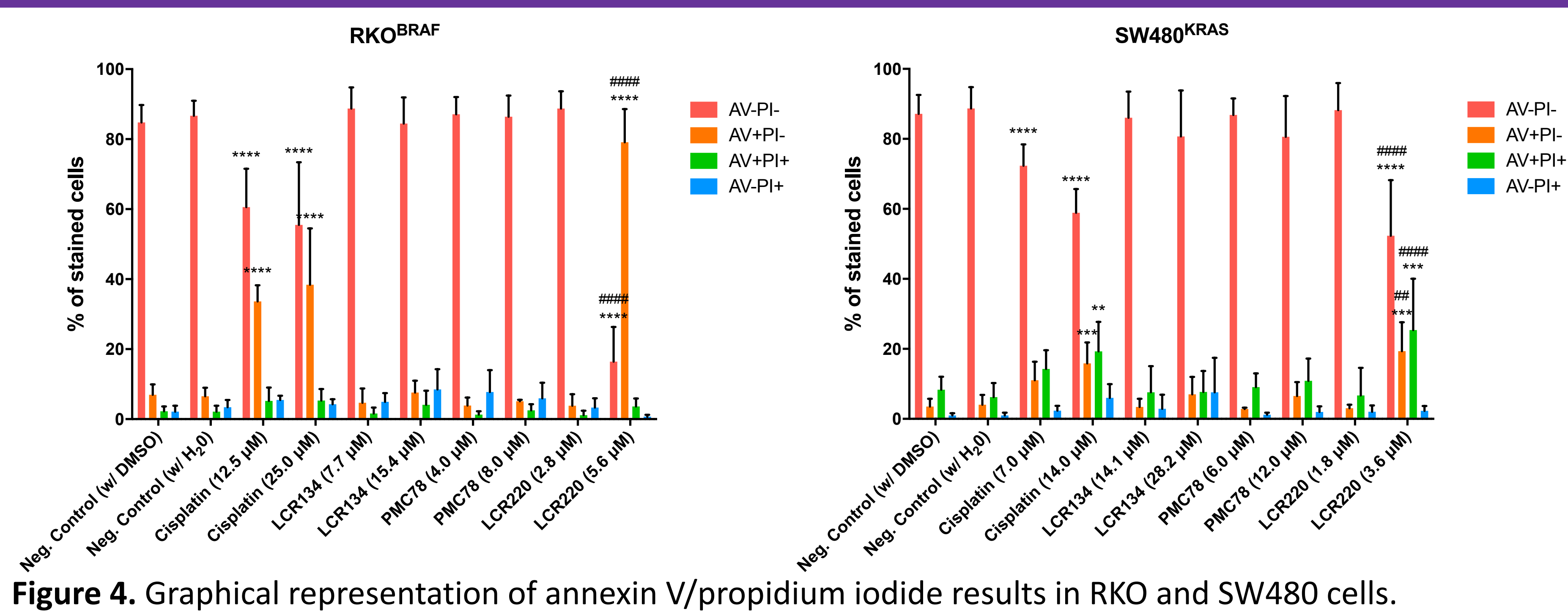

Figure 4. Graphical representation of annexin V/propidium iodide results in RKO and SW480 cells. Ru agents affect cell migration in CRC cells RKO BRAF

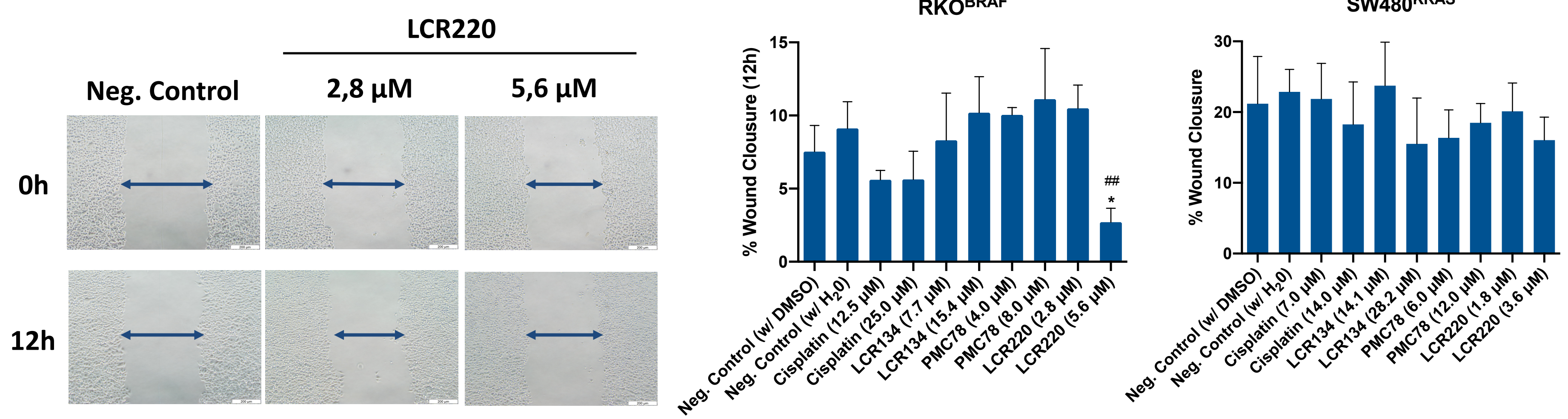

Figure 5. Representative images of RKO cells and graphical representation of wound healing assay in RKO and SW480 cells

Ru agents are located in cytoskeleton and membrane in CRC cells

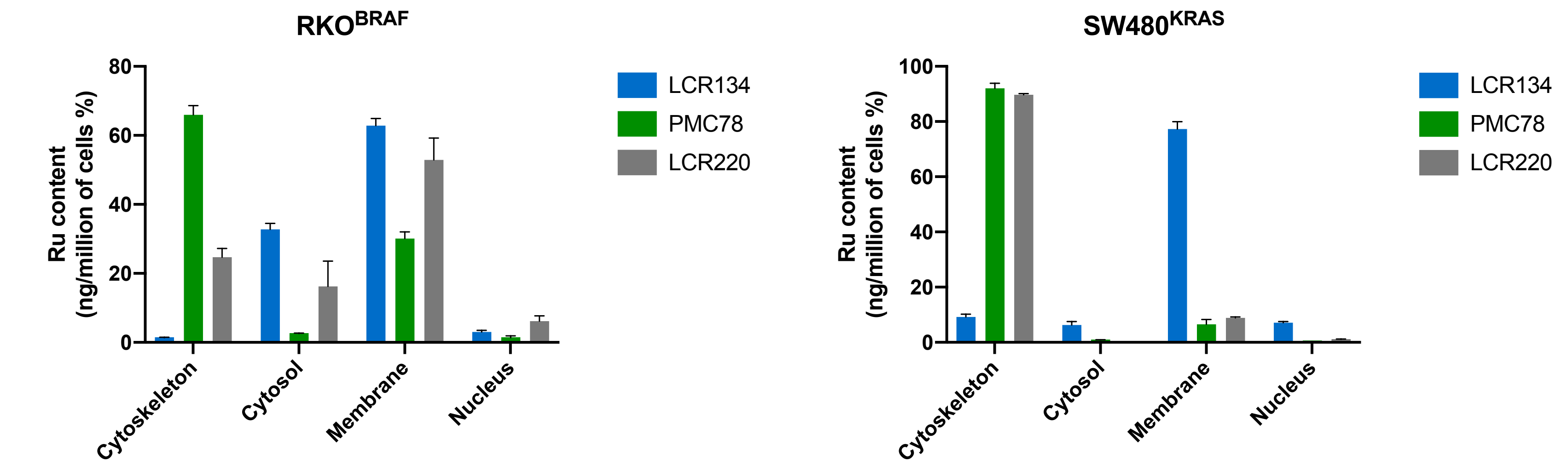

Figure 6. Graphical representation of cellular distribution of Ru agents in RKO and SW480 cells.

Ru agents affect the actin cytoskeleton of CRC cells

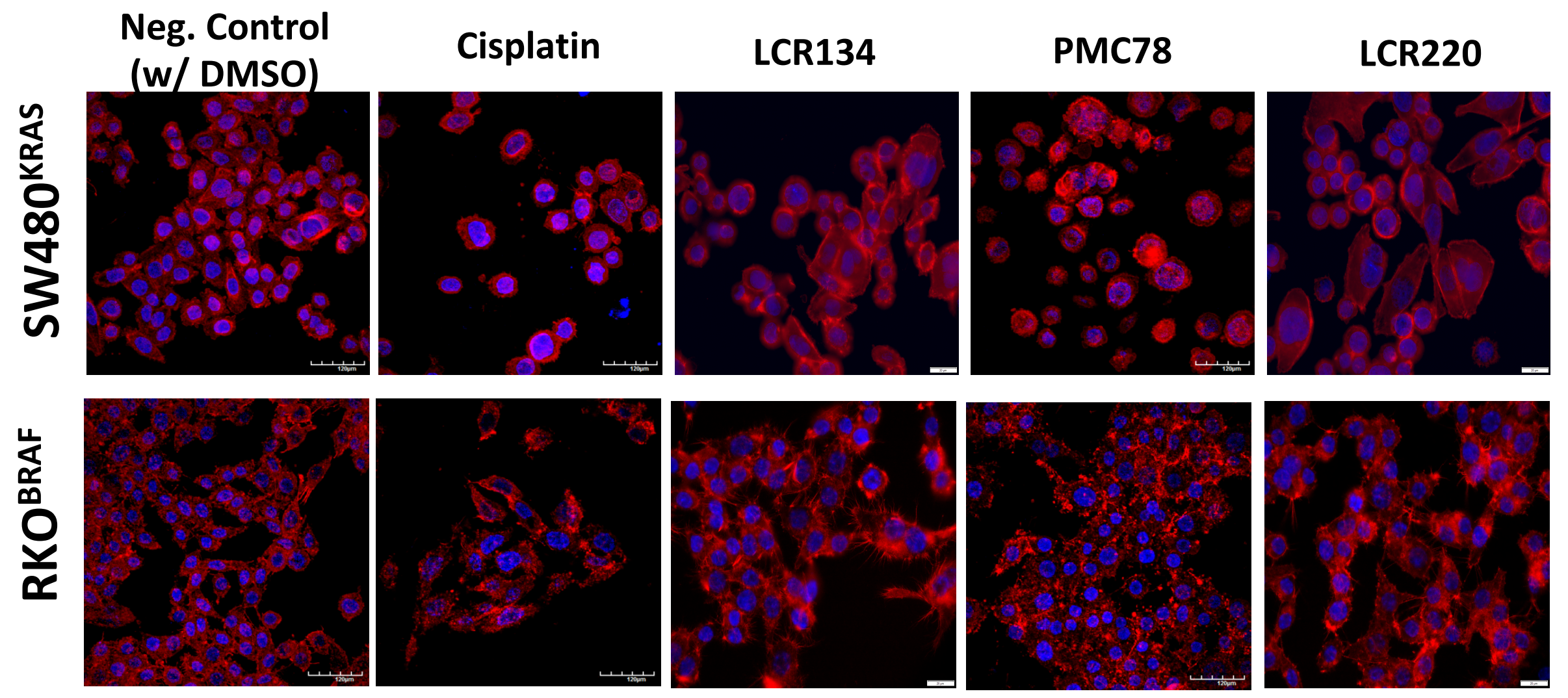

Figure 7. Analysis of F-actin staining in CRC cells. Representative images $(\times 600)$ of DAPI, Phalloidin-AlexaFluor ${ }^{\circledR} 568$

were obtained by confocal microscopy.

\footnotetext{
$\checkmark$ Ru agents show high cytotoxicity and selectivity towards CRC cells, affecting the clonogenic ability and inducing apoptosis.

$\checkmark$ Ru agents decrease motility at high concentrations and preferentially localize in membrane and cytoskeleton in CRC cells.

Ru agents also affect F-actin polymerization suggesting that actin might be a possible target for these compounds.
} 